\title{
Associations in the Two-Nucleon Transfer Reactions
}

\author{
Sajida G. Abdulvahabova ${ }^{1}$, Rasim A. Ahmedov ${ }^{2}$ and Irada G. Afandiyeva ${ }^{2}$ \\ 1. Chair "Structure of Matter", Baku State University, 23 Z.Khalilov st, Baku (AZ 1148), Azerbaijan \\ 2. Physics Chair, Azerbaijan State Oil Academy, 20 Azadliq ave, Baku (AZ1010), Azerbaijan
}

\begin{abstract}
On the basis of the association theory of nuclear structure, we have studied the ( $t, p)$ reaction. Study was carried out with the distorted plane waves of triton and of proton. It has been suggested that bineutron association is acceptable if the time during which the association maintains its structure, is large compared with the time when neutrons are in a dissociated form, and there is no exchange of nucleons between fragments associations. The cross section is written as a product of two factors, one is the spectroscopic factor which reflects the nature of the nuclear structure concerned and the other describes the process in which the target nucleus captures two nucleons as a cluster into an orbit which is characterized by a form factor. In the argument, that the radial wave function of two neutrons which form association captured nuclei close to each other, this leads to the formation of bineutron association on the nuclei surface. In this approach, the proton is emitted at the same point, which is captured bineutron association.
\end{abstract}

Key words: Scattering, matrix element, nucleon association, two-nucleon transfers, cross section.

\section{Introduction}

The two-nucleon transfer reactions have taken on great importance, because they are very sensitive to nuclear spectroscopy. The task of the proton-triton or triton-proton scattering is the simplest problem in the nucleon - nucleus interaction or interaction of a particle with a complex structure, which has been studied for a long time and its solution developed highly effective calculation schemes. A specific feature of the two-nucleon transfer reaction is that the amplitude of the transition, in general, it is the sum of the individual contributions of the two-particle, each with its phase and amplitude. The form factor consists of two parts. The first part is the overlap of the initial and final nuclear states, and the second part consists of the effective interaction between the outgoing proton and the neutrons.

To explain the mechanisms of processes with nucleon transfer, the association model is widely used [1]. According to this model, transmitted neutrons in $(t, p)$ reactions exist in the nucleus as association. Bineutron

Corresponding author: Sajida G. Abdulvahabova, professor, research field: theoretical physics. E-mail: sajida.gafar@gmail.com. association in the triton is different from free nucleons, as they are surrounded by other nucleons. This field leads to a change in the association properties compared with the free neutrons [2].

Bineutrons association is acceptable provided that the time during which the association maintains its structure is large compared to the time when the neutrons are in a dissociated form.

\section{Formulation}

Considering the reaction process $t+A \rightarrow B+p$ in the laboratory system and assuming that the momentum transfer to the nucleus $B$ can be ignored. We consider the stripping bineutrons association. Let, $\vec{r}_{p}, \quad \vec{r}_{n_{1}}$ and $\vec{r}_{n_{2}}$ the coordinates of protons and neutrons in the triton $\vec{k}_{p}$ - the wave vector of the free proton, $\vec{k}_{t}$ - wave vector of the triton.

Wave function of the initial state can be written as [3]:

$$
\Phi_{i}=\Psi_{A}(\xi) F_{t}\left(\vec{r}_{t}\right) f^{S}(\vec{r}, \vec{\rho}) \chi_{1 / 2, m_{t}}
$$

where $\Psi_{A}(\xi)$ - wave function of the nuclei $A, F_{t}\left(\vec{r}_{t}\right)$ distorted wave function of the triton, $\vec{r}_{t}$ radius vector of the centre of mass of the triton, $f^{s}(\vec{r}, \vec{\rho})$ - wave function of internal motion of the triton, $r=\left|\vec{r}_{n_{1}}-\vec{r}_{n_{2}}\right|$, 
$\vec{\rho}$ - distance from the proton to the centre of gravity of the bineutron association, $S$ indicates the spin state of the captured neutrons, $\chi_{1 / 2, m_{t}}-$ spin function of the triton. The possible angular momenta were limited by the symmetry.

Association model neglect the internal structure of the associations and effects of the Pauli principle between the nucleons in the associations which are taken into account by introducing a short range repulsion between the clusters. The orthogonality condition model and excluded state model treat the associations as elementary particles, but include effects of the Pauli principle in a more microscopic way.

The function of the final state describes the state of the nucleus $\mathrm{B}$ consisting of $\mathrm{A}$ and bineutrons association and the free movement of the proton. It can be represented as:

$$
\Phi_{f}=\Psi_{B}(\xi, \vec{R}) f\left(\vec{r}_{p}\right) \chi_{1 / 2, m_{p}}
$$

where $f\left(\vec{r}_{p}\right)$ distortion function of the proton, $\vec{R}$ - the radius vector bineutron association, $\chi_{1 / 2, m_{p}}-$ spin function of the proton.

If the spin angular momentum of the association $S=0$, then the wave function $f^{S=0}(\vec{r}, \vec{\rho})$ is a symmetric function of the radius relative distance:

$$
u^{2}=r_{12}^{2}+r_{23}^{2}+r_{13}^{2}=\frac{2}{3} r^{2}+2 \rho^{2}
$$

Matrix element of the transition from state $i$ to state $f$ can be written as follows:

$$
\begin{aligned}
& M_{i \rightarrow f}=\int f_{p}^{*}\left(\vec{r}_{p}\right) \chi_{1 / 2, m_{p}}^{*} \Psi_{B}^{*}(\xi, \vec{R}) V \Psi_{A}(\xi) F_{t}\left(\vec{r}_{t}\right) \\
& f^{s}(\vec{r}, \vec{\rho}) \chi_{1 / 2, m_{t}} d \xi d \vec{\rho} d \vec{r} d \vec{R}
\end{aligned}
$$

The aspects of two-nucleon transfer reactions that depend on nuclear structure can be isolated in structure amplitudes, which do not depend on the kinematics or scattering states of the reaction. The structure amplitudes measure the parentage and the degree to which a nuclear state possesses the particular correlations predicated by the fact that the pair is transferred to or from a nuclide which itself has definite and simple correlations among its nucleons.

Distorted function of the proton and triton are chosen as plane waves:

$$
f_{p}\left(\vec{r}_{p}\right)=e^{i \vec{k}_{p} \vec{r}_{p}}, \quad F_{t}\left(\vec{R}_{t}\right)=e^{i \vec{k}_{t} \vec{R}_{t}}
$$

We will use the approximation of zero radius [4]. In this approximation, the proton is emitted at the same point, which is captured bineutrons association. Mathematically, it comes down to choosing potential as follows:

$$
V f^{s}(\vec{r}, \vec{\rho})=W(\vec{r}) \delta(\vec{\rho})
$$

The usual way of accounting for nuclear distortions in the calculation of $f_{p}\left(\vec{r}_{p}\right)$ and $F_{t}\left(\vec{R}_{t}\right)$ in the optical model leads to depressed values theoretical cross sections, since this method does not take into account the contribution of the processes associated with incoherent rescatter-tritont on nucleons of the target nucleus $A$. This effect can be accounted for using the Glauber distorted wave. These distorted waves can be written as:

$$
\begin{aligned}
& F\left(\vec{k}_{t}, \vec{R}_{t}\right)=(2 \pi)^{-3 / 2} \exp \left(\vec{k}_{t}, \vec{R}_{t}\right) x \\
& \prod_{j=1}^{A-2}\left[1-\Gamma\left(\mathbf{b}-\mathbf{b}_{j}\right) \theta\left(\mathbf{z}-\mathbf{z}_{j}\right)\right] \chi_{m}^{t}(\mathbf{s}) \\
& f\left(\vec{k}_{p}, \vec{r}_{p}\right)=(2 \pi)^{-3 / 2} \exp \left(i \vec{k}_{p}, \vec{r}_{p}\right) \\
& \prod_{j=1}^{A}\left[1-\Gamma\left(\mathbf{b}-\mathbf{b}_{j}\right) \theta\left(\mathbf{z}-\mathbf{z}_{j}\right)\right] \chi_{m}^{p}(\mathbf{s})
\end{aligned}
$$

where $\Gamma(\mathbf{b})$ - profile function, which is a two-dimensional Fourier transform of the amplitude $f(\mathbf{q})[5]$

$$
\begin{gathered}
\Gamma(\mathbf{b})=\frac{1}{2 \pi i k} \int d^{2} q e^{-i \mathbf{q b}} f(\mathbf{q}) \\
f(\mathbf{q})=i k \int_{0}^{\infty} d b b J_{0}(q b)\left[1-e^{\left.-\frac{i}{v} \int_{-\infty}^{\infty} V\left(\mathbf{b}+\hat{k}_{i} z\right) d z\right]}\right.
\end{gathered}
$$

$\theta(z)$-discrete Heaviside function, $\mathrm{b}$ - the impact parameter.

Expanding the plane waves $F\left(\vec{k}_{t}, \vec{R}_{t}\right)$ and $f\left(\vec{k}_{p}, \vec{r}_{p}\right)$ of spherical functions and taking into account the orthogonally of the spin functions, for the transition matrix element, we obtain the expression:

$$
\begin{aligned}
& M_{i \rightarrow f}^{S=0}=\delta_{m_{t}} m_{p} \sum_{l, L} 4 \pi \sqrt{(2 l+1(2 L+1))} i^{l+l} x \\
& \int \Psi_{B}^{* S=0}(\xi, \vec{R}) \Psi_{A}(\xi) W(\vec{r}) j_{l}\left(k_{p} \vec{R}^{\prime}\right) j_{L}\left(k_{t} \vec{R}\right) d \xi d \vec{r} d \vec{R}^{\prime} d \vec{R}
\end{aligned}
$$


where $\vec{R}^{\prime}=\frac{A}{A+2} \vec{R}$.

The overlap integral $\int \Psi_{B}^{* S=0}(\xi, \vec{R}) \Psi_{A}(\xi) d \xi$ is a major factor of the matrix element. It takes into account the structure of nuclei in the initial and final states. If the energy $E_{1}$ and $E_{2}$ of the first and second neutrons are close to each other, radial functions strongly overlap and then are captured by "correlated" pairs of nucleons.

The distance $R$ between the fragments associative decay is chosen in such a way that when $R>R_{a}$ overlapping fragments nucleon densities would already be so small that we can neglect the influence of the effects on the internal antisymmetrization wave functions fragments.

Transition takes place directly from the initial to the final state transfer binucleon association, without changing the internal states of nucleons. It is assumed that the association is in a bound $\mathrm{S}$ - state. The effective cross section for the reaction $(t, p)$ can be written as:

$$
\begin{aligned}
& d \sigma=\frac{E_{t} E_{A}}{\lambda^{1 / 2}\left(m_{t}^{2}+m_{A}^{2}\right)\left(2 J_{t}+1\right)} \sum(2 \pi)^{4} \delta^{4}\left(P_{t}+P_{A}-P_{p}-P_{B}\right) X \\
& N_{0}^{2}\left|M_{i \rightarrow f}\right|^{2} d P_{p}
\end{aligned}
$$

where $E_{t}=\left(p_{t}^{2}+m_{t}^{2}\right)^{1 / 2}$ and $E_{A}=\left(\mathrm{P}_{A}^{2}+m_{A}^{2}\right)^{1 / 2}-$ energy of the incident triton $t$ and nucleus $\mathrm{A}$, respectively, $\lambda(x, y, z)=(x-y-z)^{2}-4 y z-$ kinematic function.

Zero normalization of the direct reaction $(t, p)$ is somewhat ambiguous. An accurate theoretical calculation for $N_{0}$ gives the value in the range from 200 to $900 \mathrm{MeVfm}^{2 / 3}$ [6].

Problem in solving such problems is the separation of variables, because it affects the possibility of analytical calculation of integrals over the angular variables, as well as for those variables that are not associated with the interaction of particles.

The wave functions of the residual nucleus are expanded into products of wave functions of two neutrons and the target nucleus in the $j$-j coupling scheme. For the performance of the calculation, it is convenient to transform from the $j-j$ scheme to the $L-S$ scheme.

We further assume a simple configuration for the final state, and the spin of the target take zero, $I_{A}=0$, so that $J=I_{B}$ and transforming the Clebsch-Gordan coefficients, we get

$$
\begin{aligned}
d \sigma= & \frac{2 E_{t} E_{A}}{\lambda^{1 / 2}\left(m_{B}^{2}+m_{t}^{2}\right)} \sum_{L}^{\frac{2 I_{B}+1}{2 L_{t}+1}\left[S_{B A}^{\gamma J} C_{L_{t} S J}(\gamma)\right]^{2} \times}(-)^{\mu}\left\langle\operatorname{lm} \mid L_{t} \Lambda M_{L_{t}},-\mu\right\rangle \times \\
& \times\left\langle 0, L_{t} M_{L_{t}} ; \mathbf{k}_{p}\left|M_{0 \Lambda \mu}^{S S}\right| \mathbf{k}_{t}\right\rangle\left\langle\gamma, L, 0, S, S ; \mathbf{k}_{t}, \mathbf{k}_{p}\right\rangle
\end{aligned}
$$

where

$$
\mathbf{J}=\mathbf{L}_{t}+\mathbf{S}, \quad \mathbf{I}=\Lambda+\mathbf{S}, \quad L=\mathbf{L}_{t}-\Lambda
$$

$\gamma$ denotes the other quantum numbers needed for a complete description of the configuration,

$$
M_{0 \Delta \mu}^{S S}=\sum_{i}\left\langle\gamma J M k_{t}\left|M_{i \rightarrow f}\right| k_{p}(-)^{I-M} I_{B} M_{B}\right\rangle
$$

reduced matrix element of the transition and $S_{B A}^{\gamma J}$ spectroscopic factor.

In evaluating the spectroscopic factor, on the other hand, that the contributions from shells other than the unclosed one are not negligible and in view of this circumstance, the usual convention which assumes the same radial integrals for the pairing interactions for different orbits seems to be questionable, especially for the radial integrals involving different closed shells. Generally, the radial integral involving different shells decreases gradually as the distance between two shells increases.

The wave functions of the triton still have a finite amplitude inside the nucleus, and in these cases the calculated results are sensitive to the cut-off radius, especially for a shallow optical potential. However, it seems that the cutoff results come out better than those without a cutoff for higher incident energies.

The justification of the cutoff procedure is given by the following arguments. The zero-range approximation is adopted in our calculation to simplify the integration, but this approximation is known to overestimate the contribution from the nuclear interior. 
The non-local optical potential reduces the interior contributions. It would be better to express the optical potentials and shell model potentials as non-local potentials, and the usual local potential must be regarded as only an approximation. On account of these effects, the internal contribution should be reduced considerably, and this reduction will be realized by cutoff procedure.

Eq. (13) shows that if $I_{A}=0$, then each transition characterized by a particular value $J=I_{B}$. The shapes of the differential cross sections were found to be sensitive to these parameters.

Further if we consider only $\Lambda=0$, then it follows from Eq. (14), $L=L_{t}$ and $I=S$. However, in the general case, orbital $L$ and "spin" $I$ transfers, and do not identify with the orbital $L$ and spin $S$ components in Eq. (14); if $\Lambda \neq 0$, therefore, possible that $L \neq L_{t}$ and $I \neq S$.

Fig. 1 shows the differential scattering cross section for the reaction $74 \mathrm{Ge}(\mathrm{t}, \mathrm{p}) 76 \mathrm{Ge}$. The solid line corresponds to the cross section calculated by Eq. (13) point - experimental data from [7].

We did a widely used assumption that is not considered the exchange of nucleons between the two nuclei and that the nucleons - target are not excited. Then, we were limited by the fact that the internal states of the incident $t$, departing $p$, and any intermediate associations are assumed fully symmetric

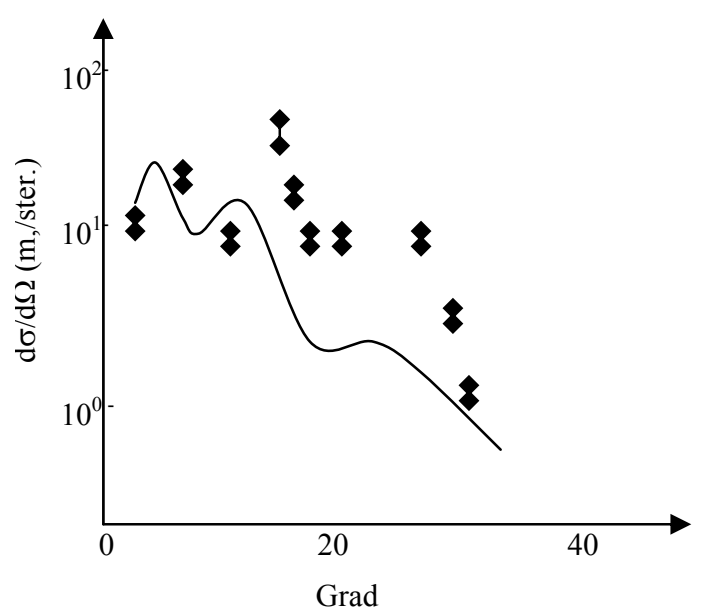

Fig. 1 The differential cross sections for the ${ }^{74} \mathrm{Ge}(t, \mathrm{p})^{76} \mathrm{Ge}$ reaction.
S-states, so that the corresponding sequential transfer interactions are diagonal in the spin states of nuclei. With this reasoning radial wave functions, two neutrons which form association captured nuclei close to one another, particularly in the peripheral region of the nucleus where there is a "cross-linking" of the wave functions of nucleons in nucleus (A) and in the nucleus B.

\section{Conclusions}

In the work, approximation of zero radius was used. For the calculation of direct processes in reactions with light associations, it would seem that we can restrict zero interaction radius. However, it is not, because even in the one-nucleon transfer reactions on light nuclei, recoil of the entire system is not small. If we consider the transfer reaction of the association, then the recoil effects are of even more importance. From this point of view, the using of zero radius interaction most justified for reactions $(d, p),(d, n)$, because recoil is less important.

When associations overlap, resonating group wave functions and shell model wave functions can be very similar after antisymmetrization. On the other hand, when associations are somewhat separated, then resonating group wave functions can include correlations which are not naturally described by shell model wave functions. It has been suggested that bineutron association is acceptable if the time during which the association maintains its structure is large compared with the time when neutrons are in a dissociated form, and there is no exchange of nucleons between fragments associations. The best wave function was found by minimizing the energy. A better wave function is obtained by projecting angular momentum and minimizing after projection. A still better approximation is to take a linear combination of these wave functions.

In the argument, that the radial wave function of two neutrons which form association captured nuclei close to each other, this leads to the formation of bineutron 
association on the nuclei surface. In this approach, the proton is emitted at the same point, which is captured bineutron association. This approach gives a good description of the scattering at high energies.

\section{References}

[1] Levin, F. S. 1987. Nucl. Phys. A. 463: 487.

[2] Berejnoy, Y. A., and Mikhayluk, V. P. 2008. "Alpha Clustering in the Processes Scattering Particles and Light Nuclei by Nuclei." PEPAN 39 (2): 437.

[3] Dubovichenko, S. B. 2004. "The Properties of Light Nuclei in the Potential Cluster Model." Almaty, edited by
Daneker, 247.

[4] Kadmensky, S. G., Kurgalin, S. D., and Chuvilsky, Y. M. 2007. "The Cluster States of Atomic Nuclei and the Processes of Cluster Decay." PEPAN 38 (6): 1333.

[5] Sitenko, A. G. 1973. "The Diffraction Scattering of Nucleons on Nuclei and Structure of Nuclei." PEPAN 4 (2):546.

[6] Pincston, W. T., and Satchler, R. 1982. "Properties of Simultaneous and Sequential Two-Nucleon Transfer." Nucl. Ph. A 383: 61-76.

[7] Becker, A. 1982. "A CCBA Description of the (p, t) Reaction to Low-Lying $0^{+}$States in the Ge Isotopes." Nucl. Ph. A 388: 477-497. 